\title{
Validación de una prueba para medir eventos vitales estresantes en adultos gay, lesbianas y bisexuales colombianos
}

\author{
Validation test in order to measure stressful life events in \\ Colombian gays, lesbians and bisexual adults
} \section{OPPicogerere}

ARTÍCULO DE
INVESTIGACIÓN
Copyright $\odot 2018$
by Psicogente

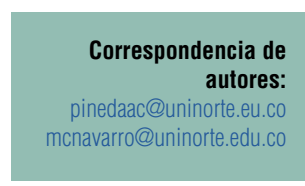

Recibido: 08-08-17 Aceptado: 22-01-18 Publicado: 08-01-19

\section{Validação de um teste para medir eventos de vida estressantes em adultos gays, lésbicas e bissexuais colombianos}

\author{
Carlos Alejandro Pineda-Roa (iD - María Camila Navarro-Segura (iD \\ Universidad del Norte, Barranquilla, Colombia
}

Resumen

Objetivo: Evaluar las características psicométricas de una prueba para valorar eventos vitales estresantes (PEVE), así como comparar el constructo según sexo, edad y orientaciones sexuales no heterosexuales residentes en tres ciudades de Colombia, Barranquilla, Bogotá y Tunja.

Método: Se implementó un muestreo no probabilístico tipo bola de nieve mediante el cual 208 participantes con edades entre 18 y 67 años $(M E=26,16 ; D E=8,56)$ diligenciaron la $P E V E$, que medía diversos eventos estresantes (EE).

Resultados: La consistencia interna obtenida a través de alfa de Cronbach fue 0,79 y el Omega de McDonald fue de 0,89 . El 34,62\% no experimentó EE durante el pasado año, el 56,25\% experimentó bajos niveles y solo un 9,13\% tuvo EE en alto grado. Análisis Paralelo y Test MAP sugieren un único factor que explicó el $54 \%$ de la varianza. Los EE en mujeres se asocian con mayor proporción de discusiones con la familia debido a su homosexualidad $(p<0,05)$, mientras que EE en hombres se asocian con problemas con la policía y ser agredidos físicamente $(p<0,05)$. Con relación a orientación sexual, EE se asocian con mayor proporción de homosexuales que reportan problemas con familiares en comparación de la proporción de bisexuales $(p<.05)$. Con respecto a la edad, adultos jóvenes tienden a experimentar mayores niveles promedio de EE que adultos $(p<.05)$.

Conclusión: En población LGB colombiana, PEVE muestra adecuada validez de constructo y consistencia interna. La mayoría de participantes perciben un nivel bajo de EE y estos niveles cambian teniendo en cuenta variables como el sexo, orientación sexual y edad. Se requieren más investigaciones en contextos latinoamericanos.

Palabras clave: Eventos vitales estresantes, Orientación sexual, Estudios de validación, Adultos colombianos.

Abstract

Objective: This paper aims to evaluate psychometric properties of the test stressful life events (SLE) and also to compare the construct according to sex, age and non-heterosexual sexual orientations in individuals who live in three different cities in Colombia, Barranquilla, Bogotá y Tunja.

Method: A non-probabilistic or snowball sampling was implemented. 208 participants between 18 and 67 years old ( $M E=26.16, S D=8.56)$ who completed the SLE test, being measured various stressful events (SE).

Results: Through Cronbach's alpha, the internal consistency was 0.79 and McDonald's Omega was 0.89 .

Cómo citar este artículo (APA):

Pineda-Roa, C.A. y Navarro-Segura, M.C. (2019). Validación de una prueba para medir eventos vitales estresantes en adultos gay, lesbianas y bisexuales colombianos. Psicogente 22(41), 1-19. https://doi.org/10.17081/psico.22.41.3305 
34.62\% did not face SE during last year, 56.25\% have experienced low levels of SE and only $9.13 \%$ faced a high degree of SE. Parallel Analysis and MAP Test suggested a single factor that showed $54 \%$ of the variance. In women, SE are associated with discussions with their family because of their homosexuality $(p<.05)$, but in men SE are associated with problems with the police and being physically assaulted $(p<.05)$. In relation with sexual orientation, $\mathrm{SE}$ are associated with homosexuals who report problems with relatives, comparing with bisexuals $(p<.05)$. According to age, results show that young adults tend to experience higher levels of $S E$ than adults $(p<.05)$.

Conclusion: In Colombian LGB population, PEVE shows adequate construct validity and internal consistency. Most participants faced low level of SE and these levels change taking into account variables such as sex, sexual orientation and age. More researches are required in Latin American contexts.

Key words: Stressful life events, Sexual orientation, Validation studies, Colombian adults.

\section{INTRODUCCIÓN}

La teoría de estrés minoritario no corresponde a una sola teoría, sino a un grupo de estudios y antecedentes de la psicología y la sociología que abordan los efectos negativos que puede tener para los individuos pertenecientes a grupos minoritarios y experimentar condiciones como el prejuicio. Algunos de ellos son los aportes de autores como Allport (1954), quien desarrolló ideas en torno a la naturaleza del prejuicio, la división endogrupo-exogrupo, la norma en el grupo, entre otros; y Durkheim (1951), quien sostuvo que el individuo necesita una regulación moral que proviene de la sociedad en la que vive para manejar sus propios anhelos y necesidades. Así mismo, se reconocen otros autores como Goffman (1963) y Link y Phelan (2001) y su conceptualización del estigma.

Con base en lo anterior, el estrés en las minorías sexuales (lesbianas, gay, bisexuales LGB) puede ser entendido a grandes rasgos como el estar en una situación o entorno en donde se contraponen los valores dominantes con los de una minoría, lo que resulta en un conflicto con el entorno por parte de los miembros de la minoría (Mirowsky \& Ross, 1989). Ahora bien, la teoría de estrés minoritario (Brooks, 1981; Meyer, 1995; Meyer, 2003) hace referencia el estrés al que se ven expuestos los individuos de categorías sociales estigmatizadas debido a su posición social desaventajada, dentro de un contexto marcadamente heterosexista.

La teoría del estrés minoritario describe procesos asociados al estrés que son particulares de la población LGB. Dentro de estos procesos o componentes se encuentran: la experiencia de eventos estresantes, la expectativa de rechazo, la homofobia internalizada y el ocultamiento de la propia condición sexual. Meyer (2003) enfatiza que estos componentes hacen parte de un continuo y 
que están ampliamente interrelacionados. El presente artículo se centra en eventos estresantes (EE) percibidos por individuos LGB.

Desde esta teoría, dichos eventos se conceptualizan como distales (externos y objetivos) y proximales o internos con respecto al individuo (Meyer, 2003; Brewster, Moradi, DeBlaere, \& Velez, 2013; Meyer, Frost \& Nezhad, 2015). Sin embargo, aunque unos tengan su origen por fuera del individuo (experiencias de prejuicio) y otros respondan a procesos psicológicos internos (expectativa de estigma, homofobia internalizada y ocultamiento de la condición sexual), la teoría del estrés minoritario reconoce que tanto los estresores distales como los proximales están mediados en algún grado por percepciones subjetivas y por el contexto sociocultural, respectivamente (Meyer, 2003).

Todos estos eventos repercuten en la salud física y mental de las personas que los experimentan. El estudio de Meyer (1995), reveló relaciones significativas entre variables como la expectativa de rechazo, las experiencias de prejuicio y la homofobia internalizada sobre el estrés psicológico, sobre todo en aquellos aspectos relacionados con ansiedad general, problemas sexuales (en el caso de la homofobia internalizada) y conducta suicida. Posterior al estudio anterior, varias aproximaciones empíricas han confirmado estos hallazgos. En efecto, Ortiz-Hernández (2005) encontró apoyo empírico a la relación entre homofobia internalizada, percepción de estigma y ocultamiento de la condición sexual, con mayor riesgo de presentar ideación e intento de suicidio, trastornos mentales y alcoholismo en población homosexual mexicana. Pineda-Roa (2016) también encontró relación entre eventos estresantes percibidos por hombres homosexuales y bisexuales y homofobia internalizada.

Así mismo, estudios como los de Huebner y Davis (2007) encuentran correlaciones positivas entre la percepción de discriminación de hombres homosexuales y bisexuales y la cantidad de días que han estado enfermos en el lapso de un año. Ehlers, Zuyderduin y Oosthuizen (2001) resaltan la relación positiva entre el bienestar de personas LGB y la aceptación interna y externa (social) de la orientación sexual. Por otro lado, se ha establecido en estudios como los de Lock y Steiner (1999) que al comparar poblaciones de adolescentes heterosexuales y LGB, este último grupo muestra más altos niveles de conductas de afrontamiento que son útiles de sobrellevar el estrés y las dificultades asociados con el estatus de diversidad sexual. 
Estudios similares han encontrado apoyo empírico a la relación entre eventos estresantes relacionados con minorías sexuales y salud como por ejemplo las experiencias de homofobia y conductas autodestructivas (McDermott, Roen, \& Scourfield, 2008); la homofobia internalizada, la expresión de la identidad sexual, la discriminación laboral y su relación con la calidad de relaciones amorosas en mujeres lesbianas (Barrantes, Eaton, Veldhuis, \& Hughes, 2017); la expresión u ocultamiento de la identidad sexual y el sentimiento de vergüenza, culpa y su afectación en la salud de hombres homosexuales (Bybee, Sullivan, \& Zielonka, 2009); estrés minoritario y problemas sexuales (Zamboni \& Crawford, 2007); discriminación percibida y salud física (Huebner \& Davis, 2007).

En 1993, Rosario, Hunter y Gwadz diseñaron una prueba publicada en el año 2002, que incluye 12 ítems que miden diversos eventos estresantes relacionados con ser lesbiana/gay/bisexual (Rosario, Hunter \& Gwadz, 1993; Rosario, Schrimshaw, Hunter, \& Gwadz, 2002). Posteriormente en un proyecto colaborativo, J. Toro y A. Urzúa propusieron una versión de 10 ítems de la misma prueba, traducida y adaptada por un equipo de trabajo de la Universidad de Puerto Rico, la cual no ha sido publicada a la fecha. El presente trabajo presenta los datos correspondientes a una muestra de varones y mujeres autoidentificados como LGB en Colombia, de dicha versión de la PEVE de diez ítems en español. En el anexo se detallan los ítems de la prueba.

En Colombia, aún no se ha indagado sobre el estrés que perciben las minorías sexuales, específicamente debido al estigma asociado a su orientación sexual. Además, en el 2013, Meyer y Frost propusieron que "investigadores deben desarrollar más medidas centradas en su modelo de estrés minoritario". Por tanto, en ausencia de instrumentos válidos y confiables para población sexualmente diversa en Colombia, que den cuenta de dichos eventos estresantes, el objetivo del presente estudio es reportar las propiedades psicométricas de una prueba de eventos vitales estresantes (PEVE) en población LGB residente en tres ciudades colombianas.

\section{MÉTODO}

\subsection{Diseño}

La presente investigación es de carácter psicométrico de tipo instrumental, debido a que está encaminada al desarrollo de pruebas y aparatos, inclu- 
yendo tanto el diseño o adaptación, como el estudio de sus propiedades psicométricas (Montero \& León, 2007).

\subsection{Participantes}

Se implementó un muestreo no probabilístico tipo bola de nieve mediante el cual, participaron 208 individuos autoidentificados según escala Kinsey (Kinsey, Pomeroy, \& Martin, 1948) en donde se establecen diferentes grados de comportamientos sexuales. En este estudio se toman en cuenta ocho categorías en un continuo de " 0 " a "7" correspondientes a "atracción exclusiva por mujeres", "atracción principal por mujeres y solo incidental por hombres", "atracción principalmente por mujeres y más que incidental por los hombres", "igual atracción por hombres y mujeres", "atracción principal por hombres y más que incidental por mujeres", "atracción principalmente por hombres y solo incidentalmente por mujeres", "atracción exclusiva por hombres" y "no estoy seguro".

Participaron 63 mujeres lesbianas (30,3\%) y 145 hombres gay $(69,7 \%)$, con edades comprendidas entre los 17 y 67 años ( $M E=26,34, D E=8,72)$. Todos los participantes residían en tres ciudades colombianas, dos del interior de Colombia, Bogotá ( $n=73)$, Tunja ( $n=49)$, y Barranquilla $(n=85)$, esta última ubicada en la región Caribe colombiana. De acuerdo a las puntuaciones en la escala Kinsey, 94 participantes se identificaron como principal o exclusivamente homosexuales (incluye mujeres lesbianas y hombres gay) correspondientes al $45,2 \%$ y 114 como bisexuales de ambos sexos que corresponden a $54,8 \%$. Ningún participante puntuó 0 ni 7 en dicha escala.

\subsection{Instrumento}

Los participantes diligenciaron la prueba de eventos estresantes PEVE, compuesta por 10 ítems con puntuación dicótoma, en la que "sí" era presencia de estrés y "no" era ausencia del mismo en el último año. PEVE arroja puntajes entre 0 y 10, a mayor puntaje mayor nivel de EE. Los puntajes totales de EE se categorizaron en tres grandes rangos siendo 5 el punto medio teórico sugerido que divide a quienes tienen bajo y alto $E E$, y 0 para ausencia de $E E$.

La PEVE fue traducida y adaptada en Puerto Rico por el Dr. José Toro y este se aplicó originalmente en una muestra de varones homosexuales latinoamericanos en un proyecto denominado Estudio latinoamericano de bienestar psicológico, calidad de vida y riesgo de trastornos de alimentación en varones 
gay de diez países, liderado por Toro y Urzúa de la Universidad de Puerto Rico y la Universidad Católica del Norte en Chile respectivamente, cuya muestra en Colombia fue recolectada por el primer autor del presente trabajo.

\subsection{Procedimiento}

El estudio se llevó a cabo en varias fases. Primeramente, se contactaron líderes de centros donde concurrían personas LGB en las tres ciudades. Una vez autorizado el acceso a la población se solicitó el diligenciamiento del consentimiento informado. Seguidamente se realizó una primera aproximación a los participantes con una prueba piloto en la que participaron 20 personas LGB. El objetivo del pilotaje era verificar la validez aparente (validez del usuario a quien va dirigida la prueba), y comprobar que los ítems se comprendían completamente. Los resultados del pilotaje sugirieron cambios en las instrucciones del instrumento, refinando las propuestas por Rosario et al. (2002). Por ejemplo, se preguntó por la ocurrencia de eventos estresantes debidos a su homosexualidad/bisexualidad en el último año y no en los últimos tres meses. Las nuevas instrucciones pueden verse en la sección Anexo. Posteriormente se llevó a cabo la aplicación definitiva y se elaboró una base de datos en SPSS versión 22.

\subsection{Análisis de datos}

El estadístico de Kaiser Meyer Olkin (KMO) y Test de esfericidad de Bartlet permitieron establecer la factibilidad del Análisis Factorial Exploratorio (AFE). La consistencia interna fue medida a través de alfa de Cronbach y Omega de McDonald ( $(2)$. La discriminación de los ítems fue calculada mediante el coeficiente de correlación biserial puntual corregida. Análisis Paralelo, y Test de MAP fueron calculados mediante el Software Factor versión 10.3.01 (Lorenzo Seva \& Ferrando, 2006) y permitieron confirmar el número de factores a retener. Dado que el constructo $\mathrm{EE}$ no se distribuye normalmente, validez discriminante se evaluó mediante pruebas de comparación de dos grupos independientes por medio de $U$ de Mann Whitney con el fin de establecer diferencias estadísticamente significativas.

\subsection{Conflictos de interés}

Los autores del artículo declaran que no existe ningún interés comercial, asociativo o de otro tipo que presente un conflicto de intereses con este trabajo. 


\section{RESULTADOS}

A continuación, se presentan los resultados obtenidos, primero mediante el análisis descriptivo en términos de frecuencias y porcentajes del EE, seguido de los estadísticos descriptivos de los ítems de la PEVE, así como la caracterización de los EE según las variables sexo, orientación sexual y edad. Luego se presentan los resultados de confiabilidad por consistencia interna a través del Alfa de Cronbach y Omega de McDonald, por último, validez de constructo y discriminante.

\subsection{Resultados descriptivos y discriminación de los ítems}

Puntuaciones totales de estrés señalan que el 34,62\% no experimentó EE durante el último año, el $56 \%$ tuvo bajos niveles de EE y solo un 9,13\% experimentó EE en alto nivel.

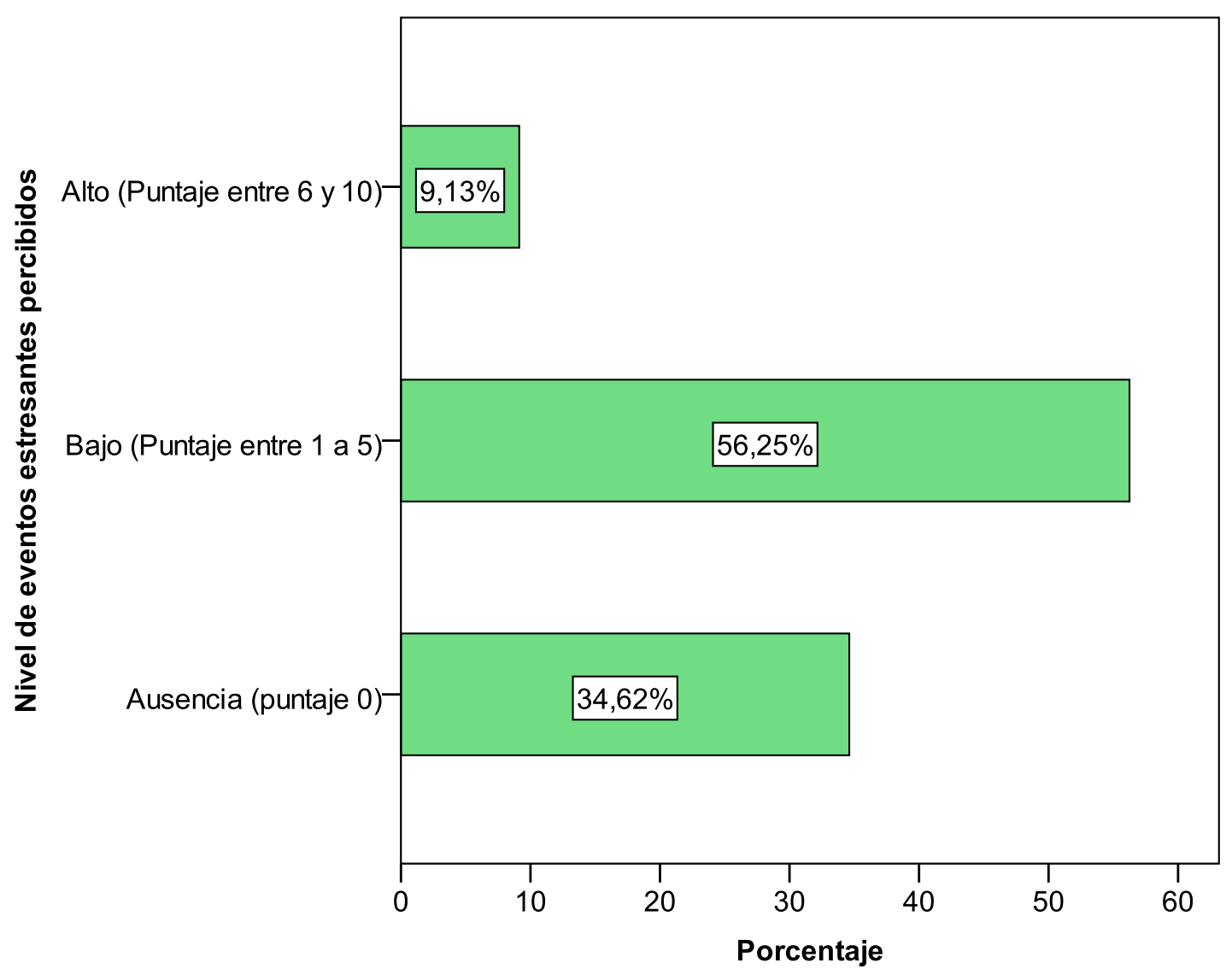

Figura 1. Distribución porcentual de eventos estresantes

La Figura 1 señala que la mayoría de participantes perciben un nivel bajo de estrés. La Tabla 1 señala que todos los ítems de la PEVE discriminan 
adecuadamente en tanto que la correlación ítem total corregida siempre fue superior a 0,30 , indicando que hay una relación entre la percepción de estrés y obtención de un puntaje alto en la prueba.

Tabla 1.

Estadísticas de los ítems de la PEVE

\begin{tabular}{|c|c|c|c|c|c|c|}
\hline & \multicolumn{2}{|c|}{ SI } & \multicolumn{2}{|c|}{ NO } & \multirow{2}{*}{$\begin{array}{l}\text { CORRELA- } \\
\text { CIÓN BISERIAL } \\
\text { PUNTUAL } \\
\text { CORREGIDA }\end{array}$} & \multirow{2}{*}{$\begin{array}{l}\text { ALFA DE CRON- } \\
\text { BACH SI EL ÍTEM SE } \\
\text { HA SUPRIMIDO }\end{array}$} \\
\hline & $\mathbf{N}$ & $\%$ & $\mathbf{N}$ & $\%$ & & \\
\hline $\begin{array}{c}\text { Aumento del número } \\
\text { de discusiones entre } \\
\text { sus padres debido a su } \\
\text { homosexualidad }\end{array}$ & 76 & 36,5 & 132 & 63,5 & 0,61 & 0,76 \\
\hline $\begin{array}{c}\text { Aumento de problemas } \\
\text { con sus hermanos o } \\
\text { hermanas debido a sus } \\
\text { homosexualidad }\end{array}$ & 36 & 17,3 & 172 & 82,7 & 0,54 & 0,77 \\
\hline $\begin{array}{l}\text { Aumento del núme- } \\
\text { ro de desacuerdos o } \\
\text { discusiones entre Ud. y } \\
\text { sus padres debido a su } \\
\text { homosexualidad }\end{array}$ & 80 & 38,5 & 128 & 61,5 & 0,56 & 0,76 \\
\hline $\begin{array}{l}\text { Incremento del número } \\
\text { de desacuerdos o discu- } \\
\text { siones con otros miem- } \\
\text { bros de su familia debido } \\
\text { a su homosexualidad }\end{array}$ & 57 & 27,4 & 151 & 72,6 & 0,56 & 0,76 \\
\hline $\begin{array}{l}\text { Problemas con profeso- } \\
\text { res/as o sus jefes/as por } \\
\text { su homosexualidad }\end{array}$ & 21 & 10,1 & 187 & 89,9 & 0,35 & 0,79 \\
\hline $\begin{array}{l}\text { Problemas con sus } \\
\text { compañeros/as de } \\
\text { clase o compañeros/as } \\
\text { de trabajo debido a su } \\
\text { homosexualidad }\end{array}$ & 36 & 17,3 & 171 & 82,6 & 0,43 & 0,78 \\
\hline $\begin{array}{l}\text { Perdida de amigos/as } \\
\text { cercanos debido a su } \\
\text { homosexualidad }\end{array}$ & 39 & 18,8 & 169 & 81,3 & 0,47 & 0,78 \\
\hline $\begin{array}{l}\text { Incremento del número } \\
\text { de discrepancias o } \\
\text { discusiones con amigos/ } \\
\text { as cercanos debido a su } \\
\text { homosexualidad }\end{array}$ & 36 & 17,3 & 172 & 82,7 & 0,42 & 0,78 \\
\hline $\begin{array}{l}\text { Problemas con la } \\
\text { policía debido a su } \\
\text { homosexualidad }\end{array}$ & 26 & 12,5 & 182 & 87,5 & 0,30 & 0,79 \\
\hline $\begin{array}{l}\text { Ser asaltado o agredido } \\
\text { físicamente en un inci- } \\
\text { dente o ataque debido a } \\
\text { su homosexualidad }\end{array}$ & 26 & 12,5 & 182 & 87,5 & 0,37 & 0,79 \\
\hline
\end{tabular}




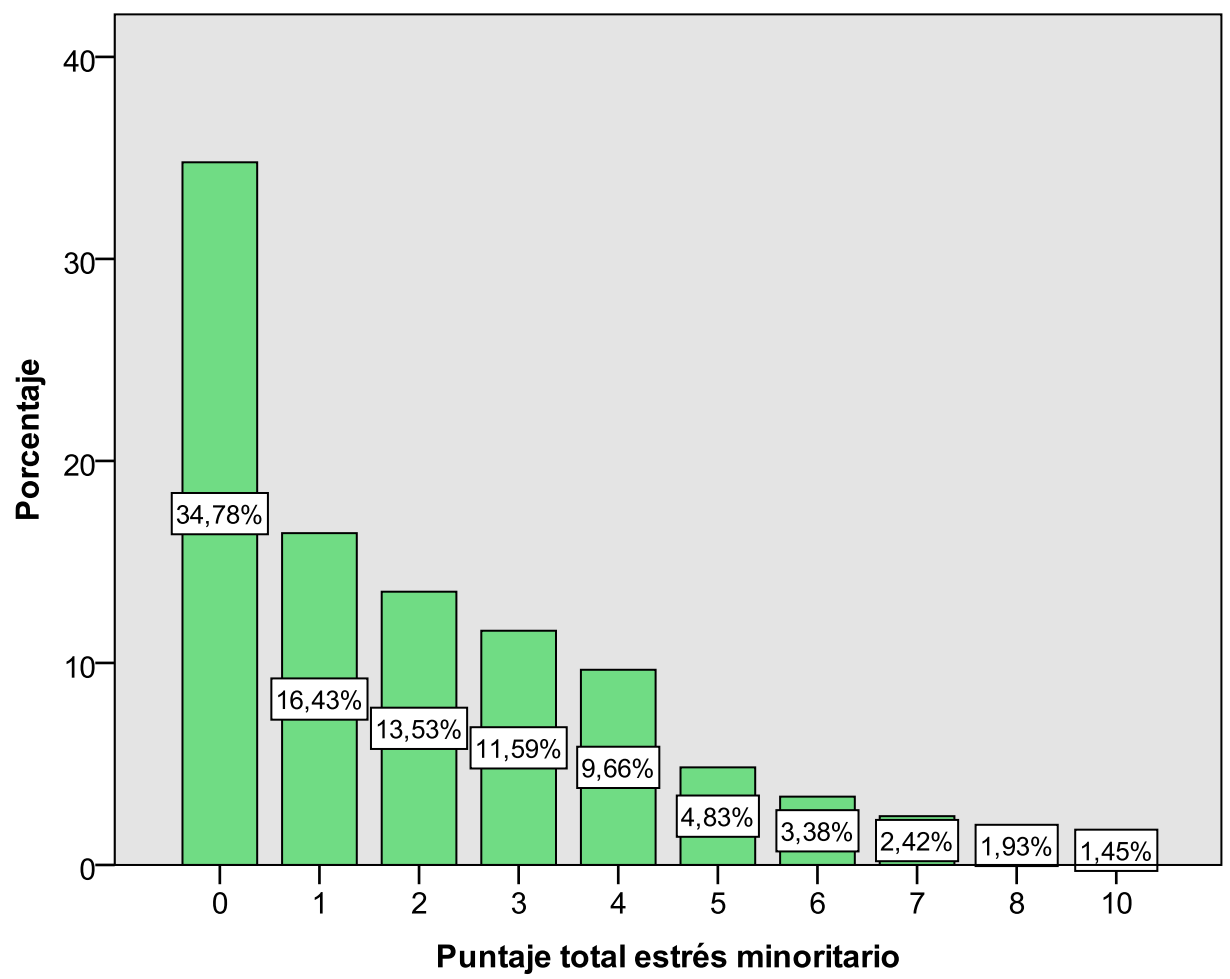

Figura 2. Distribución porcentual del estrés percibido entre el mínimo y el máximo esperado

Igualmente, todos los ítems contribuyen a la confiabilidad. La Figura 2 pone de manifiesto la distribución de los porcentajes de individuos entre el mínimo puntaje total teórico esperado (0) y el máximo puntaje teórico esperado (10). Claramente la distribución del atributo EE es asimétrica positiva $(A s=1,23)$. Uno de cada tres participantes no percibe estrés debido a su orientación sexual.

\subsection{Resultados de consistencia interna}

Los resultados obtenidos mediante Alfa de Cronbach arrojaron un valor de 0,79 y de 0,89 para el Omega de McDonald, valores que están dentro de un rango adecuado de confiabilidad.

\subsection{Resultados de validez de constructo}

El estadístico de Bartlet $(465,6 ; \mathrm{gl}=45 ; \mathrm{p}=0,00)$ y la prueba $\operatorname{KMO}(0,76)$ sugieren que un análisis factorial es adecuado. A través de la regla de Kaiser de auto valores mayores que uno, AFE calculado mediante el método de extracción factorización de eje principal y rotación Promax sugirió tres factores 
(estresores familiares, sociales y de violencia). No obstante, debido a que la regla de Kaiser tiende a sobredimensionar el número de factores, se aplicó un análisis paralelo basado en el método de Análisis de Factor de Rango Mínimo (Timmerman \& Lorenzo-Seva, 2011), el cual permitió confirmar la existencia de un único factor, que explicó el 54,3\% de la varianza y que denominamos eventos estresantes (EE) (Tabla 2). Allí se presentan los pesos factoriales de cada ítem que oscilaron entre 0,34 y 0,72. Estos valores nos permiten inferir que la escala no es tau-equivalente y que, por tanto, la medida de confiabilidad Omega de McDonald es más adecuada.

Tabla 2.

Resultado Análisis Paralelo

\begin{tabular}{ccccc}
\hline ÍtEM & $\begin{array}{c}\text { DATO REAL } \\
\text { \% VARIANZA }\end{array}$ & $\begin{array}{c}\text { MEDIA ALEATORIA \% } \\
\text { VARIANZA }\end{array}$ & $\begin{array}{c}\text { PERCENTIL 95 ALEATORIO } \\
\text { \% VARIANZA }\end{array}$ & PESOS FACTORIALES \\
\hline 1 & $54,3^{*}$ & 21,2 & 24,3 & 0,72 \\
2 & 13,4 & 17,8 & 20,3 & 0,67 \\
3 & 10,7 & 15,1 & 17,1 & 0,60 \\
4 & 8,0 & 12,8 & 14,5 & 0,56 \\
5 & 5,0 & 10,7 & 12,2 & 0,48 \\
6 & 3,4 & 8,7 & 10,2 & 0,47 \\
7 & 2,7 & 6,7 & 8,4 & 0,44 \\
8 & 2,0 & 4,6 & 6,3 & 0,37 \\
9 & 0,4 & 2,4 & 4,3 & 0,36 \\
\hline
\end{tabular}

* Número de dimensiones recomendadas: 1

El método MAP (por sus siglas en inglés Minimum Average Partial) de Velicer (1976), conocido como Test MAP, también sugiere retener un único factor. El gráfico de sedimentación permite una visión del factor encontrado (Figura 3).

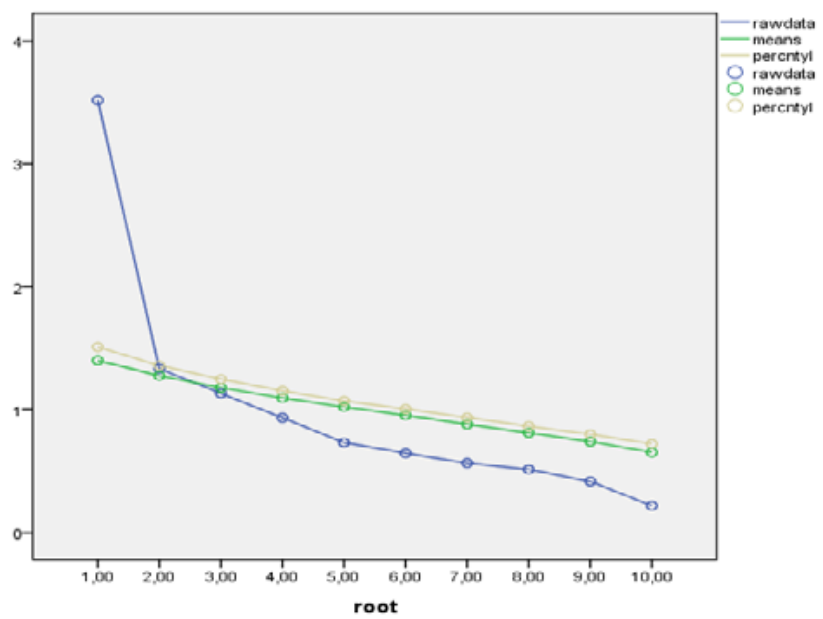

Figura 3. Gráfico de sedimentación de la PEVE 


\subsection{Resultados de validez discriminante}

La validez discriminante se refiere al poder en que una prueba discrimina o diferencia entre las subpoblaciones a las cuales les fue aplicada. Se observa si el constructo eventos estresantes discrimina según sexo, orientación sexual y edad. Como se aprecia en la Tabla 3, EE en mujeres se asocian con mayor proporción de desacuerdos o discusiones con sus padres y otros familiares debido a su homosexualidad $(p<0,05)$, mientras que EE en hombres se asocian con problemas con la policía y ser asaltados o agredidos físicamente en un incidente $o$ ataque $(p<0,05)$. En los demás ítems no se encontraron diferencias importantes.

\section{Tabla 3.}

Diferencias de EE según la variable sexo del participante

\begin{tabular}{ccccc}
\hline & $\begin{array}{c}\text { AUMENTO DEL NÚME- } \\
\text { RO DE DESACUERDOS O } \\
\text { DISCUSIONES ENTRE UD. } Y \\
\text { SUS PADRES DEBIDO A SU } \\
\text { HOMOSEXUALIDAD }\end{array}$ & $\begin{array}{c}\text { INCREMENTO DEL NÚMERO } \\
\text { DE DESACUERDOS O DISCU- } \\
\text { SIONES CON OTROS MIEM- } \\
\text { BROS DE SU FAMILIA DEBIDO } \\
\text { A SU HOMOSEXUALIDAD }\end{array}$ & $\begin{array}{c}\text { PROBLEMAS } \\
\text { CON LA POLICIA } \\
\text { A CAUSA DE SU } \\
\text { HOMOSEXUALIDAD }\end{array}$ & $\begin{array}{c}\text { SER ASALTADO O AGREDIDO } \\
\text { FÍSICAMENTE EN UN INCI- } \\
\text { DENTE O ATAQUE DEBIDO A } \\
\text { SU HOMOSEXUALIDAD }\end{array}$ \\
\hline Mujeres (n) & 23 & 17 & 0 & 3 \\
$\%$ & 53,48 & 39,53 & 0 & 6,97 \\
Hombres(n) & 37 & 29 & 20 & 20 \\
$\%$ & 28,68 & 22,48 & 15,50 & 15,50 \\
$p$ & 0,0006 & 0,009 & 0,0013 & 0,07 \\
\hline
\end{tabular}

Con relación a la variable orientación sexual, resultados arrojaron un rango promedio superior de EE en homosexuales en comparación con el rango promedio de EE en la muestra de bisexuales $(p<0,05)$.

Tabla 4.

Diferencias según orientación sexual y rangos de edad en la PEVE

\begin{tabular}{|c|c|c|c|c|c|}
\hline & & $\mathbf{N}$ & RANGO PROMEDIO & $\begin{array}{l}\text { SUMA DE } \\
\text { RANGOS }\end{array}$ & $\underset{\text { MANN-WHITNEY }}{U}$ \\
\hline \multirow{3}{*}{ Orientación sexual } & Homosexualidad (Kinsey 5, 6) & 93 & 119,89 & 11149,50 & \multirow{3}{*}{$3823,500 *$} \\
\hline & & & & & \\
\hline & Bisexualidad (Kinsey 2, 3 y 4) & 114 & 91,04 & 10378,50 & \\
\hline \multirow{3}{*}{ Grupo etario } & Adultos (Mayor de 30 años) & 51 & 72,81 & 3713,50 & \multirow{3}{*}{$2387,500^{*}$} \\
\hline & & & & & \\
\hline & Adultos jóvenes (entre 18 y 29 años) & 156 & 114,20 & 17814,50 & \\
\hline & Total & 207 & & & \\
\hline
\end{tabular}


Con respecto a la edad, tabla 4 señaló que adultos jóvenes tienden a experimentar mayores niveles promedio de EE que sus contrapartes adultos $(p<0,05)$.

\section{DISCUSIÓN}

El objetivo del presente estudio fue revisar las propiedades psicométricas de una prueba para medir EE en población LGB residente en tres ciudades colombianas. En materia de propiedades psicométricas de la prueba en cuestión, los resultados obtenidos mediante Alfa de Cronbach y el Omega de McDonald arrojaron valores que están dentro de un rango adecuado de confiabilidad ( $\alpha=0,79, \Omega=0,80$ ). Una versión similar de la PEVE de Rosario et al. 2002 arrojó un $\alpha$ de 0,80, (Rotheram-Borus et al., 1995).

En cuanto a validez de constructo, a pesar de haber encontrado inicialmente tres factores, métodos más robustos como el Análisis Paralelo y Test de MAP, los cuales son independientes del tamaño muestral, permitieron corroborar la existencia de un único factor. Además de lo anterior, ítems discriminan adecuadamente, en tanto que correlación ítem-total fue superior a 0,30 , indicando que la prueba discrimina entre quienes puntuaron afirmativamente a cada ítem y obtuvieron un puntaje alto. En consecuencia, PEVE posee consistencia interna y validez de constructo adecuada en adultos LGB.

A partir de los resultados del presente estudio, pudo establecerse que dos de cada tres participantes perciben algún nivel de estrés debido a su orientación sexual. Sin embargo, se observan algunas diferencias en lo que respecta al estrés percibido, cuando se analiza el sexo, la edad y la orientación sexual de los participantes. Los EE en mujeres se asociaron con una mayor proporción de discusiones con sus padres y otros miembros de la familia debido a su homosexualidad y los EE en hombres se asociaron con problemas con la policía y con ser agredidos físicamente en un incidente o ataque. Es decir, la violencia está asociada a estresores en hombres gay. Lo anterior encuentra respaldo en estudios como el de Ortiz-Hernández (2005), en donde se afirma que las mujeres lesbianas y bisexuales del estudio mostraron porcentajes más altos de respuestas que incluían reacciones negativas por parte de la familia hacia una mujer lesbiana en comparación con los hombres bisexuales y homosexuales. Paradójicamente, también se ha encontrado en otros estudios, que la apertura o expresión de la propia orientación sexual hacia la familia o entorno social, se ha relacionado con mejor salud mental en las mujeres pertenecientes a minorías sexuales, en comparación con sus contrapartes masculinas (Kuyper \& Fokkema, 2011). 
Así mismo, los estudios de Baams, Grossman y Russell (2015) y McDaniel, Purcell y D’Augelli (2001), muestran que los hombres son más propensos a vivir experiencias de victimización por motivo de su orientación sexual; en parte porque estos transgreden de manera más significativa o evidente los comportamientos asignados socialmente al rol del hombre, por lo que son comúnmente sujetos de violencia y acoso (McDaniel, Purcell \& D’Augelli, 2001).

En ese sentido, la realidad que hombres gais, mujeres lesbianas y bisexuales viven no puede generalizarse por completo, ya que cuenta con particularidades relacionadas con componentes sociales que van configurando la dinámica de relación entre personas con diferentes orientaciones sexuales. En todo caso estos componentes sociales que incluyen generalmente estereotipos impulsan consecuencias importantes (Calvo, 2013) que afectan tanto a hombres como a mujeres (Hequembourg \& Brallier, 2009). En ese mismo sentido, Campo-Arias, Herazo, y Oviedo (2017), sostienen que el prejuicio hacia hombres gais y mujeres lesbianas tiene una alta correlación, lo que sugiere que son dos aspectos que no son totalmente distintos, al menos al ser medidos con escalas específicas. Sin embargo, la vivencia y experiencia del que es receptor del prejuicio, puede presentar diferencias según la orientación sexual como se observa en este estudio.

Por otro lado, cuando se analiza la relación entre EE, sexo y orientación sexual de los participantes del presente estudio, se observa que los EE se asocian con un menor rango promedio de estrés percibido en bisexuales en comparación con el rango promedio de estrés percibido en la población homosexual. Baams, Grossman y Russell (2015), tuvieron resultados similares en su estudio. Encontrando que las mujeres bisexuales reportaron menos niveles de estrés por motivo de revelar su orientación sexual, al ser comparados con hombres gais y hombres bisexuales. Así mismo, resultados como los del estudio de Kuyper y Fokkema (2011) muestran que aquellos participantes LGB que presentaban mayores niveles de homofobia internalizada y reacciones negativas de otras personas por motivo de su orientación sexual reportaban más problemas a nivel de salud mental, siendo los hombres homosexuales y las mujeres lesbianas quienes tienen una salud mental más pobre en comparación con las personas bisexuales.

Algunos estudios como los de Brewster et al. (2013), introducen el tema de la flexibilidad cognitiva para explicar esta diferencia entre bisexualidad y homosexualidad. Los autores encontraron que los bisexuales puntuan mas 
alto en flexibilidad cognitiva que los homosexuales. Según ellos, las personas bisexuales "se mueven entre contextos heteronormativos y contextos de marginalización gay y lésbico", lo que refleja su flexibilidad en relación a nociones binarias de la orientación e identidad sexual (Charyton, 2007; Konik \& Crawford, 2004).

Por otro lado, el estudio de Baams et al. (2015) en los Estados Unidos, reportó que los participantes de más edad percibían niveles más altos de estrés minoritario en cuanto a variables como victimización por orientación sexual y estrés por ocultamiento de la orientación sexual. Por su parte, en el presente estudio fueron los adultos jóvenes los que tendieron a experimentar mayores niveles promedio de EE que los adultos. Esto refleja diferencias importantes en el contexto colombiano en comparación con el estadounidense y nos acerca a entender que la naturaleza de los EE no puede verse alejada del contexto social y geográfico. En efecto, también se encontró que entre mayor tamaño poblacional de la ciudad, menor era la percepción de EE. No obstante, este resultado no fue significativo.

Es importante señalar algunas limitaciones. Primero, la implementación de un muestreo no probabilístico que no permite establecer generalizaciones más allá de las personas LGB de las muestras de las tres ciudades colombianas. Segundo, el presente estudio no indagó la correlación entre los EE y otros constructos de salud mental. Por tanto, futuras investigaciones deberán establecer la validez convergente y divergente, con el fin de aportar más evidencia a la validez de la PEVE. Tercero, es necesario realizar estudios en donde se pueda determinar el poder predictivo y no solo asociativo de los EE con otros constructos de la salud física y mental de las minorías sexuales. Por último, un estudio multivariado podría dar luces sobre las variables que modulan y moderan el estrés de minorías, con el fin de evaluar la utilidad en la reducción de los síntomas del estrés debido al estigma asociado a la orientación sexual. Por tanto, dada la naturaleza unidimensional de la PEVE hallada en el presente estudio, se recomienda aplicarla junto con otras pruebas como el afrontamiento, apoyo social y otras medidas de salud mental propias de las minorías sexuales.

Futuras investigaciones en Latinoamérica deberán corroborar o refutar los presentes hallazgos, debido a que la investigación en países de habla hispana es muy escasa, lo que hace difícil la comparación con literatura previa en contextos culturales similares a los de Colombia. Algunos participantes señalaron que no habían revelado su orientación sexual y que por 
tanto, la prueba no aplicaba para ellos. No obstante, hemos sugerido nuevas instrucciones que permitan que cualquier persona no heterosexual pueda responder los ítems, independientemente del nivel de coming out en el que se encuentre. Así, si alguien no ha salido del closet, es muy probable que no haya tenido discusiones, problemas o discrepancias debido a su orientación sexual, por lo que, para dichas personas la respuesta a los ítems de la prueba será No. No obstante, el ocultamiento de la orientación sexual no implica ganancia sino por el contrario, una condición de salud más precaria para la población LGB, conforme lo han evidenciado muchos estudios previos (Ardila, 2008; Meyer, 1995; 2003; Meyer \& Frost, 2013; Ortiz-Hernández, 2005), en los que se recomienda que los terapeutas afirmativos procuren la autoaceptación de la orientación sexual de sus consultantes.

A pesar de que el estrés es un concepto central en la psicología contemporánea, muy pocas investigaciones se han llevado a cabo sobre EE en minorías sexuales en Latinoamérica. Quizá una de las razones sea los importantes avances jurídicos y legales que se han dado en países de habla hispana incluido Colombia, como son la Ley Antidiscriminación en el 2011, el logro del Matrimonio Igualitario en el 2016 y la adopción por parejas del mismo sexo en el 2015 , en donde la realidad de las minorías sexuales ha visto grandes avances sociales en el reconocimiento de sus derechos. Sin embargo, aún queda camino por superar el estigma y la homofobia que propician el estrés.

Se concluye que la PEVE posee propiedades psicométricas adecuadas y que puede ser usada por clínicos e investigadores en la valoración de los EE en contextos colombianos similares a los aquí descritos. Se requieren estudios de seguimiento diferenciales para cada subgrupo dentro del colectivo LGB que corroboren las diferencias encontradas en el presente estudio y que puedan evidenciar si realmente el estrés de minorías está en descenso en Colombia.

Financiamiento: El presente trabajo hace parte del proyecto de investigación "Determinantes individuales y contextuales del riesgo de suicidio en jóvenes autoidentificados según orientación sexual" financiado mediante la convocatoria 727 de Colciencias.

Agradecimientos: Los autores agradecen a Colciencias por financiación de su Doctorado en Psicología, Universidad del Norte.

\section{REFERENCIAS}

Allport, G. W. (1954). The nature of prejudice. Reading, MA: Addison-Wesley. 
Ardila, R. (2008). Homosexualidad y Psicología. (2 ed.). Bogotá: El Manual Moderno.

Baams, L., Grossman, A., \& Russell, S. (2015). Minority stress and mechanisms of risk for depression and suicidal ideation among lesbian, gay, and bisexual youth. Developmental Psychology, 51,(5), 688-696. http://dx.doi.org/10.1037/a0038994

Barrantes, R., Eaton, A., Veldhuis, C., \& Hughes, T. (2017). The Role of Minority Stressors in Lesbian Relationship Commitment and Persistence Psychology of Sexual Orientation and Gender Diversity The Role of Minority Stressors in Lesbian Relationship. Psychology of Sexual Orientation and Gender Diversity, 4(2), 1-13. http://doi.org/10.1037/sgd0000221

Brewster, M. E., Moradi, B., DeBlaere, C., \& Velez, B. L. (2013). Navigating the Borderlands: The Roles of Minority Stressors, Bicultural Self-Efficacy, and Cognitive Flexibility in the Mental Health of Bisexual Individuals. Journal of Counseling Psychology, 60(4), 543-556. Doi: http://doi.org/10.1037/a0033224

Brooks, V. R. (1981). Minority stress and lesbian women. Lexington, MA: Lexington Books

Bybee, J. A., Sullivan, A. E. L., \& Zielonka, A. E. (2009). Are Gay Men in Worse Mental Health than Heterosexual Men? The Role of Age, Shame and Guilt, and Coming-Out. Journal Adult Development, 16, 144-154. http://doi.org/10.1007/ s10804-009-9059-x

Calvo, Y. (2013). Homoparentalidad: explorando el reconocimiento social y los derechos de los homosexuales en la ciudad de San Luis, Argentina. Psicogente, 16(29), 118-131. http://revistas.unisimon.edu.co/index.php/psicogente/article/ view/1945

Campo-Arias, A., Herazo, E. \& Oviedo, H. (2017). Correlación entre actitud hacia homosexualidad femenina y masculina en estudiantes de Medicina. Psicogente, 20(37), 172-180. http://doi.org/10.17081/psico.20.37.2426

Charyton, C. (2007). What Is the Relationship Between Sexual Orientation, Bisexuality and Creativity? Journal of Bisexuality, 6(4), 49-69. http://doi.org/10.1300/ J159v06n04_05

Durkheim, E. (1951). Suicide: A Study in Sociology. New York: Che Free Press.

Ehlers, V. J., Zuyderduin, A., \& Oosthuizen, M. J. (2001). The well-being of gays, lesbians and bisexuals in Botswana. Issues and Innovations in Nursing Practice, 35(6), 848-856. https://doi.org/10.1046/j.1365-2648.2001.01922.x

Goffman, E. (1963). Stigma: Notes on the management of spoiled identity. New York: Touchstone.

Hequembourg, A. L., \& Brallier, S. A. (2009). An exploration of sexual minority stress across the lines of gender and sexual identity. Journal of homosexuality, 56(3), 273-298. https://doi.org/10.1080/00918360902728517

Huebner, D. M., \& Davis, M. C. (2007). Perceived Antigay Discrimination and Physical Health Outcomes. Health Psychology, 26(5), 627-634. http://doi. org/10.1037/0278-6133.26.5.627

Konik, J., \& Crawford, M. (2004). Exploring normative creativity: Testing the relationship between cognitive flexibility and sexual identity. Sex Roles, 51(3), 249-253. https://doi.org/10.1023/B:SERS.0000037885.22789.83

Kinsey, A., Pomeroy, W., Martin, C. (1948). Sexual behavior in the human male. Philadelphia, Pa: WB Saunders Co.

Kuyper, L., \& Fokkema, T. (2011). Minority stress and mental health among dutch LGBs: Examination of differences between sex and sexual orientation. Journal of Counseling Psychology, 58(2), 222-233. https://doi.org/10.1037/a()022688 
Link, B. G., \& Phelan, J. C. (2001). Conceptualizing stigma. Annual Review of Psychology, 27, 363-85. https://doi.org/0360-0572/01/0811-0363

Lock, J., Ph, D., \& Steiner, H. (1999). Relationships between Sexual Orientation and Coping Styles of Gay, Lesbian, and Bisexual Adolescents from a Community High School. Journal of the Gay and Lesbian Medical Association, 3(3), 77-82. https:// doi.org/10.1023/A:10222359107493.

Lorenzo-Seva, U., \& Ferrando, P. J. (2006). FACTOR: A computer program to fit the exploratory factor analysis model. Behavior research methods, 38(1), 88-91. https://doi.org/10.3758/BF03192753

McDaniel, J., Purcell, D., \& D'Augelli, A. (2001). The relationship between sexual orientation and risk for suicide: Research findings and future directions for research and prevention. Suicide and Life-Threatening Behavior, 31, 84-105. https://doi. org/10.1521/suli.31.1.5.84.24224

Mcdermott, E., Roen, K., \& Scourfield, J. (2008). Avoiding shame: young LGBT people, homophobia and self-destructive Avoiding shame: young LGBT people, homophobia and self-destructive behaviours. Culture, Health \& Sexuality, 10(8), 851-829. https://doi.org/10.1080/13691050802380974

Meyer, I.H. (1995). Minority stress and mental health in gay men. Journal of Health and Social Behavior, 36(1), 38-56. https://doi.org/10.2307/2137286

Meyer, I. H. (2003). Prejudice, social stress, and mental health in lesbian, gay, and bisexual populations: Conceptual issues and research evidence. Psychological Bulletin, 129, 674-697. http://doi.org/10.1037/0033-2909.129.5.674

Meyer, I.H., \& Frost, D. (2013). Minority Stress and the Health of Sexual Minorities. In C. J. P. and A. R. D'Augelli (Ed.), Handbook of Psychology and Sexual Orientation (First Edition, Vol. 6, pp.252-266). Oxford University: Oxford University. https:// doi.org/10.1093/acprof

Meyer, I.H., Frost, D.M., \& Nezhad, S. (2015). Minority Stress and Suicide in Lesbians, Gay Men, and Bisexuals. In: P. Goldblum, D. L. Espelage, J. Chu, \& B. Bongar (Eds.), Youth Suicide and Bullying: Challenges and Strategies for Prevention and Intervention (pp.177-187). New York: Oxford University Press.

Mirowsky, J., \& Ross, C. E. (1989). Social causes of psychological distress. New York: Aldine de Gruyter.

Montero, I., \& León, O. G. (2007). Sistema de clasificación del método en los informes de investigación en Psicología. International Journal of clinical and health psychology, 5(1). Health and Social Behavior, 36, 38-56. https://doi. org/10.2307/2137286556

Ortiz-Hernández, L. (2005). Influencia de la opresión internalizada sobre la salud mental de bisexuales, lesbianas y homosexuales de la Ciudad de México. Salud Mental, 28(4), 49-65. http://www.scielo.org.mx/scielo.php?script=sci_arttext\&pid=S0185-33252005000400049

Pineda Roa, C. A. (2016). Propiedades psicométricas de una prueba de homonegatividad internalizada en varones homosexuales y bisexuales colombianos. Psicología desde el Caribe, 33(1), 47-65. https://dx.doi.org/10.14482/psdc.33.1.8074

Rosario, M., Hunter, J., \& Gwadz, M. (1993). Gay-Related Stressful Life Events Measure. Unpublished instrument.

Rosario, M., Schrimshaw, E. W., Hunter, J., \& Gwadz, M. (2002). Gay-related stress and emotional distress among gay, lesbian, and bisexual youths: A longitudinal examination. Journal Consulting Clinical Psychology, 70(4), 967-975. https://doi. org/10.1037//0022-006X.70.4.000 
Rotheram-Borus, M. J., Reid, H., Gillis, R., Rotheram-Borus, M. J., Reid, H., Insti-, N., Koopman, C. (1995). Prevalence, Course, and Predictors of Multiple Problem Behaviors Among Gay and Bisexual Male. Developmental Psychology, 31(1), 75-85. https://dx.doi.org/10.1037/0012-1649.31.1.75

Timmerman, M. E., \& Lorenzo-Seva, U. (2011). Dimensionality Assessment of Ordered Polytomous Items with Parallel Analysis. Psychological Methods, 16(2), 209-220. https://dx.doi.org/10.1037/a0023353

Velicer, W. F. (1976). Determining the number of components from the matrix of partial correlations. Psychometrika, 41, 321-327. https://dx.doi.org/10.1007/ BF02293557

Zamboni, B. D., \& Crawford, I. (2007). Minority Stress and Sexual Problems among African-American Gay and Bisexual Men. Archives of Sexual Behavior, 36, 569-578. https://doi.org/10.1007/s10508-006-9081-z 


\section{ANEXO \\ Prueba de eventos vitales estresantes (PEVE)}

\section{INSTRUCCIONES}

A continuación encontrará una lista de cosas que le suceden a la gente. Por favor, díganos en cada caso si esta le ha ocurrido o no en el último año debido a su homosexualidad/bisexualidad. Cada una de las diez afirmaciones se compone de dos partes, por ejemplo, en el ítem 5, la primera parte: problemas con sus profesores o jefes y la segunda parte: debido a su homosexualidad/bisexualidad. Para contestar Sí, deben cumplirse o ser ciertas las dos partes. Basta con que una de las dos partes no se cumpla o no aplique para que su respuesta sea NO. Por ejemplo, si usted, nunca ha revelado su orientación sexual a sus familiares, claramente no habrá tenido discusiones con ellos debido a esta.

\begin{tabular}{ccc}
\hline & ÍtEM & NO \\
\hline 2 & Aumento del número de discusiones entre sus padres debido a su homosexualidad \\
3 & Aumento de problemas con sus hermanos o hermanas debido a su homosexualidad \\
4 & Incremento del número de desacuerdos o discusiones con otros miembros de su familia debido a su \\
5 & Promosexualidad \\
6 & Problemas con sus compañeros/as de clase o compañeros/as de trabajo debido a su homosexualidad \\
7 & Pérdida de amigos/as cercanos debido a su homosexualidad \\
9 & Incremento del número de discrepancias o discusiones con amigos/as cercanos debido a su homosexualidad \\
10 & Problemas con la policía debido a su homosexualidad \\
\hline
\end{tabular}

